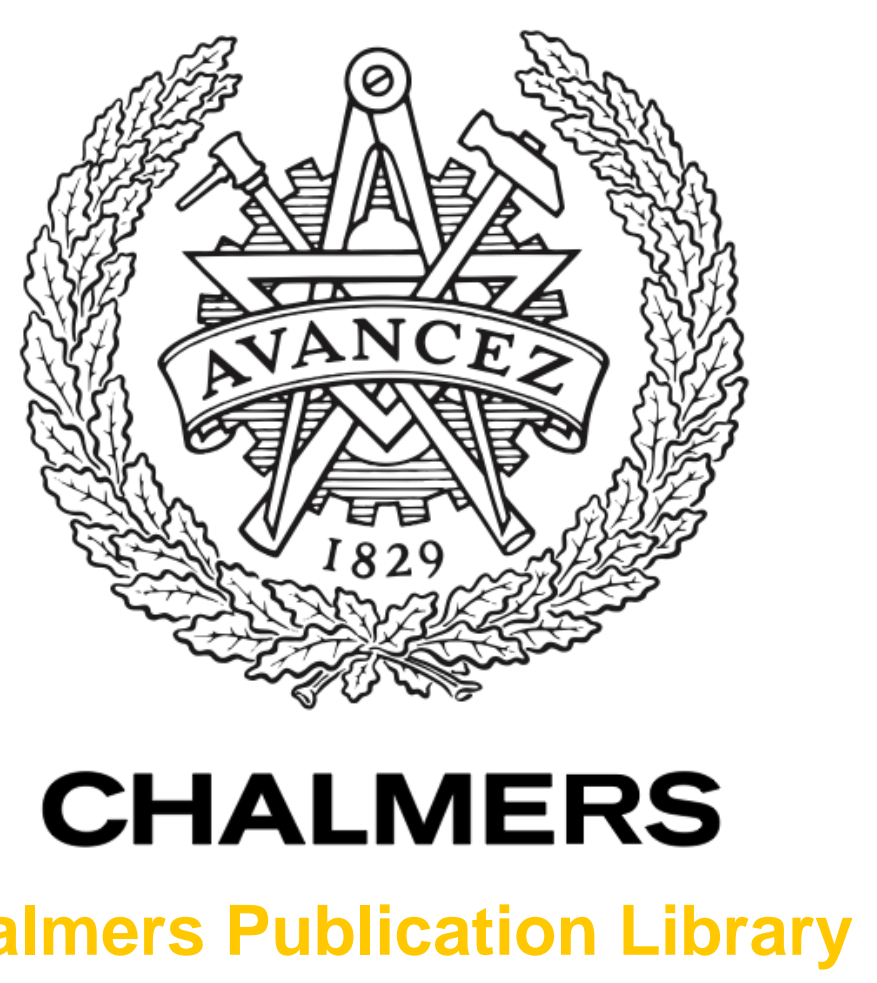

Simultaneous Thermal and State-of-Charge Balancing of Batteries: A Review

This document has been downloaded from Chalmers Publication Library (CPL). It is the author's version of a work that was accepted for publication in:

IEEE Vehicle Power and Propulsion Conference, 27-30 October 2014, Coimbra Portugal

Citation for the published paper:

Altaf, F. ; Johannesson, L. ; Egardt, B. (2014) "Simultaneous Thermal and State-of-Charge Balancing of Batteries: A Review". IEEE Vehicle Power and Propulsion Conference, 27-30

October 2014, Coimbra Portugal

Downloaded from: http://publications.lib.chalmers.se/publication/206640

Notice: Changes introduced as a result of publishing processes such as copy-editing and formatting may not be reflected in this document. For a definitive version of this work, please refer to the published source. Please note that access to the published version might require a subscription. 


\title{
Simultaneous Thermal and State-of-Charge Balancing of Batteries: A Review
}

\author{
Faisal Altaf, Lars Johannesson and Bo Egardt
}

\begin{abstract}
The battery pack lifetime is severely affected by the State-of-Charge (SOC) and thermal imbalance among its cells, which is inevitable in large automotive batteries. In this review paper, the need of simultaneous thermal and SOC balancing is emphasized. Thermal and SOC balancing are two tightly coupled objectives. However, we argue here that it is possible to achieve these simultaneously by using a balancing device that enables the non-uniform use of cells, optimally using the brake regeneration phases and load variations in the drive cycle, and exploiting cell redundancy in the battery pack. The balancer must provide extra degree-of-freedom in control by distributing a large battery pack into smaller units to enable an independent cell/module-level control of a battery system.
\end{abstract}

\section{INTRODUCTION}

A cell is the smallest packaged form of a battery. A battery sub-module (BSM) is a collection of two or more cells connected in series, battery module (BM) is a collection of two or more BSMs connected in series or parallel, and a battery pack (BP) is a collection of several BMs connected in series and parallel to meet voltage, energy and power requirements. The battery pack, containing long strings of series-connected cells, is one of the most expensive components in the powertrain of battery powered electrified vehicles (xEVs). As a result, the battery lifetime is an important factor for the success of $\mathrm{xEVs}$. The lithium-ion battery, due to its relatively higher specific energy and long deep cycle-life, is surfacing as one of the major alternative choices for future xEVs. However, like all other cell chemistries, the ageing rate of each Li-ion cell in a battery pack is greatly affected by various factors like the state-of-charge (SOC) level, depth-of-discharge (DOD), and temperature of each cell. The cells in the string being stored or cycled at higher SOC-level, DOD and temperature age faster than those at lower SOC, DOD, and temperature [1], [2], [3], [4], [5], [6]. Thus, thermal, SOC, and DOD imbalance among cells would cause nonuniform ageing of battery packs. The analysis of nonuniform ageing in Li-ion packs is given in [7].

The SOC imbalance in the cell strings is primarily caused by variations in cell actual capacities, leakage currents, and operating conditions whereas thermal imbalance is mainly caused by variations in cell internal resistance and temperature gradient in the coolant, which is not negligible in the battery packs of xEVs [8], [9], [10], [11]. The SOC and capacity imbalance also leads to DOD imbalance, which affects the cycle life of a cell [3]. Although, small variations in cell characteristics are inevitable due to manufacturing tolerances, but these variations enhance further with time due to nonuniform ageing of cells. This situation is aggravated further because

The authors are with Chalmers University of Technology, Department of Signals and Systems, 41296 Gothenburg Sweden. E-mails: ffaisal.altaf, larsjo,bo.egardt\}@chalmers.se. The work of the authors was supported by the Chalmers Energy Initiative. of tight coupling between the cell imbalance and nonuniform ageing which may result in a vicious cycle: imbalance causes nonuniform ageing which in turn causes even more imbalance and so on. If this cycle continues, it may severely affect the performance of a battery pack, resulting in significant reduction of its lifetime due to premature failure of only one cell in the string, regardless of the high state-of-health of other cells. In addition, the SOC imbalance also has a detrimental impact on the total capacity of the BSM. In short, the total capacity of unbalanced BSM is a function of initial cell SOCs, which may vary a lot and, therefore, greatly reduce the total capacity of a BSM. Thus, thermal and SOC balancer is very critical for optimal performance of automotive batteries.

The SOC balancing can be achieved using various types of passive or active SOC balancers, see [12], [13], [14], [15], [16], whereas thermal balancing can potentially be achieved using active cooling with reciprocating air-flow (RF), see [10]. The notion of simultaneous thermal and SOC balancing was introduced first, according to the best of our knowledge, in our previous work, see [17], [18], and [19]. Thermal and SOC balancing are two tightly coupled and somewhat conflicting objectives. In addition to reviewing the negative impact of thermal and SOC imbalance on battery ageing, the main contribution of this paper is that it adds more thorough discussion and insight to the simultaneous balancing problem through some simple arguments and illustrations, without going into any mathematical intricacies. We show the possibility of achieving these coupled objectives using a single active balancer working based on a load sharing concept. In particular, some specific requirements for such an integrated balancing device are discussed and then cascaded h-bridge multilevel converter (CHB MLC) is presented as one possible option in this regard. In addition, we also discuss some specific characteristics of the load profile (drive cycle) required for achieving these objectives.

The paper is organized as follows. Section II and III reviews the impact of thermal and SOC imbalance on the lithiumion battery ageing and the total capacity of a BSM. Some typical SOC and thermal balancing methods are reviewed in sections IV and V. Section VI discusses the feasibility of simultaneous thermal and SOC balancing. Some specific requirements on the load profile and the balancing hardware are also discussed in this regard. In section VII, the feasibility of simultaneous balancing is illustrated through a simple simulation-based case study for a series string of two cells. Finally, section VIII concludes the paper.

\section{IMPACT OF SOC IMBALANCE}

The SOC imbalance has a detrimental impact not only on the BSM ageing but also on its total capacity. In this section, 
these two aspects will be discussed in detail.

\section{A. Reduction of BSM Capacity}

The capacity and SOC of a BSM is a function of cell SOCs and their capacities. Let us consider an unbalanced BSM consisting of a series string of $n$ cells, Cell ${ }_{1}$ to Cell $_{n}$, with different capacities $\left(C_{1} \cdots C_{n}\right)$ and SOCs $\left(\xi_{1} \cdots \xi_{n}\right)$. Let us also assume that all the cells have equal leakage current. Since the BSM has no balancing device, it will have SOC variations among its cells all the time. In this case, the charging is stopped when any cell in the string reaches its fully charged state (or its EOCV) and similarly the discharging is stopped when any cell in the string reaches its fully discharged state (or its EODV). The chargeable capacity of an unbalanced BSM, with unequal cell capacities, is given by [20], [21], [22]

$$
C_{\mathrm{BSM}, C}^{u}(t)=\min _{i}\left(\left(1-\xi_{i}(t)\right) C_{i}\right)
$$

and its remaining dischargeable capacity is given by

$$
C_{\mathrm{BSM}, D}^{u}(t)=\min _{i}\left(\xi_{i}(t) C_{i}\right) .
$$

Thus, the maximum possible capacity of the unbalanced BSM at any time $t$ is given by

$$
C_{\mathrm{BSM}}^{u}(t)=C_{\mathrm{BSM}, D}^{u}(t)+C_{\mathrm{BSM}, C}^{u}(t)
$$

and its $S O C$ is given by

$$
\xi_{\mathrm{BSM}}^{u}(t)=\frac{C_{\mathrm{BSM}, D}^{u}(t)}{C_{\mathrm{BSM}}^{u}(t)}
$$

The total capacity and SOC of the BSM given by eq. (3) and eq. (4) respectively can not be easily related to the total capacity and SOC of any single cell in the BSM. Thus, in order to simplify the expressions, let us assume the BSM to be fully charged $\left(\xi_{\mathrm{BSM}}^{u}=1 \Rightarrow C_{\mathrm{BSM}, C}^{u}=0\right)$ and define

$$
C_{\min }=\min _{i}\left\{\xi_{i} C_{i} \mid \xi_{\mathrm{BSM}}^{u}=1\right\}
$$

Now, we can see that the total capacity and SOC of the BSM depends entirely on the capacity and SOC of a certain $\mathrm{Cell}_{k}$ that has minimum dischargeable (chargeable) capacity in the whole string at fully charged (discharged) state of the BSM. Let us consider a simple example of unbalanced BSM shown in Fig. 1. It contains two cells with equal self-discharge currents, but Cell 1 has higher capacity than $\mathrm{Cell}_{2}$. The BSM is depicted in the fully charged and fully discharged state on the right and left hand sides of the figure respectively. During charging, Cell ${ }_{1}$ will hit its fully charged state before $\mathrm{Cell}_{2}$ and the BSM cannot be charged further for safety reasons, despite of available chargeable capacity of $\mathrm{Cell}_{2}$. This implies under-utilization of $\mathrm{Cell}_{2}$. Similarly, during discharge process, as soon as $\mathrm{Cell}_{2}$ will hit the fully discharge state, BSM will stop discharging despite of remaining dischargeable capacity in $\mathrm{Cell}_{1}$ and hence will result in its under-utilization. Thus, none of these cells are fully utilized during charge-discharge cycle. Note that $C_{\min }$, shown in the figure, is a function of SOC and capacity imbalance. Greater the SOC imbalance, less will be $C_{\min }$ and thus less will be BSM capacity. Thus, SOC imbalance can greatly reduce the total capacity of a BSM.

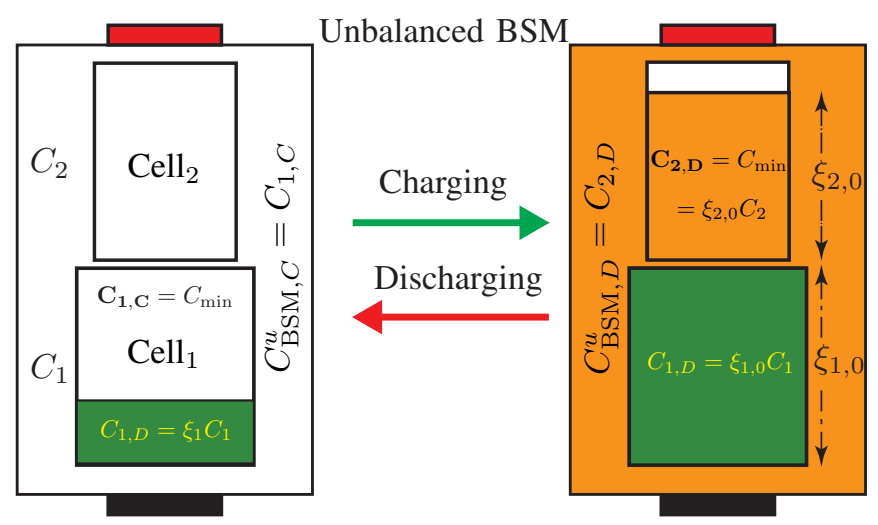

Fig. 1. Unbalanced BSM: Illustration of the impact of SOC imbalance. Note variations in cell capacities, initial cell SOCs, and the DODs.

\section{B. Non-uniform Ageing of BSM}

As shown in Fig. 1, cells in the unbalanced BSMs cycle at different DODs $\left(\mathrm{DOD}_{1}=0.68<\mathrm{DOD}_{2}=0.85\right)$. In addition, each cell starts its cycle at different initial SOC-level. Since DOD and SOC-level affects the cell ageing rate, cells of unbalanced BSMs suffer from nonuniform ageing. As the lifetime of the BSM is upper bounded by the fastest cell ageing rate in the string, therefore the unbalanced BSM may reach its end-of-life sooner due to the weakest link in the chain.

\section{IMPACT OF THERMAL IMBALANCE}

Temperature is an important factor that strongly affects the lifetime of cells. Higher the temperature of a cell, higher will be the capacity and power fading rates [3]. Thus, thermal imbalance is another major problem in large BSMs, which also needs special attention, see [8], [9], and [10] for a detailed review of thermal issues in lithium-ion batteries of $\mathrm{xEVs}$.

\section{A. Causes of Thermal Imbalance}

There are two main sources of thermal imbalance:

Variation in Cell Resistances: Fresh cells, even from the same batch, may differ in their ohmic resistance due to manufacturing tolerances. When these cells are serially connected to form a BSM, the difference may increase further with time due to nonuniform rate of resistance growth.

Temperature Gradient in the Coolant: Consider an aircooled BSM consisting of $n$ series connected cells. Thermal network diagram [10] of the BSM is shown in Figure 2. Each temperature node $T_{f i}$ models the temperature of coolant fluid element adjacent to $\operatorname{Cell}_{i}$. The temperature $T_{f i-1}$ of the upstream fluid node ' $i-1$ ' of $\mathrm{Cell}_{i}$ is related to the temperature $T_{f i}$ of downstream fluid node ' $i$ ' of $\mathrm{Cell}_{i}$ by

$$
T_{f i}=\frac{\left(T_{s i}+\beta_{\mathrm{i}} T_{f i-1}\right)}{\alpha_{\mathrm{i}}}
$$

where $\alpha_{i}=R_{u i} c_{f}, \beta_{i}=1-\alpha_{i}, R_{u i}$ is convection thermal resistance for $\mathrm{Cell}_{i}$, and $c_{f}$ is thermal conductance of the coolant. Given that $T_{f 0}$ is a known quantity, then by a forward recursion of eq. (6), any $T_{f i}$ can be expressed as a weighted sum of inlet fluid temperature $T_{f 0}$ and the temperatures $T_{s 1}$ to $T_{s i}$ of $\mathrm{Cell}_{1}$ to $\mathrm{Cell}_{i}$ respectively, as shown below.

$$
T_{f i}=a_{f i 1} T_{s 1}+a_{f i 2} T_{s 2}+\cdots+a_{f i i} T_{s i}+b_{f i} T_{f 0}
$$




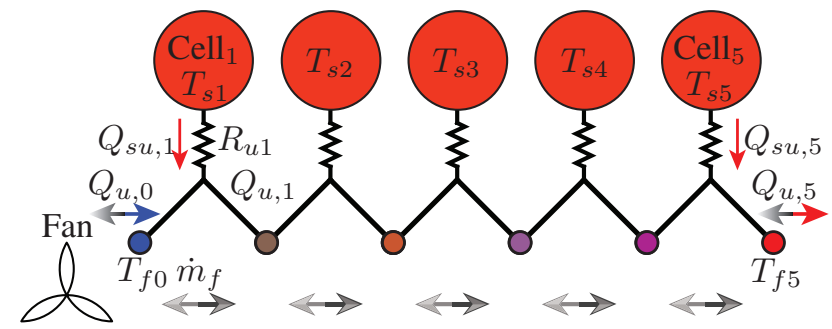

Fig. 2. Battery thermal network diagram where $Q_{s u, i}$ and $Q_{u, i}$ are heat transfer rates from $\mathrm{Cell}_{i}$ and at coolant fluid node ' $i$ ' respectively. Heat balance for left-to-right coolant flow is given by $Q_{u, i}=Q_{u, i-1}+Q_{s u, i}$

where each weight $a_{f i j}$ and $b_{f i}$ is a function of $\alpha_{i}$ and $\beta_{i}$. Thus, according to the above equation, the coolant in the BSM suffers from a temperature gradient due to build up of additive heat from cells along the coolant stream. It results in higher ambient temperature for downstream cells. Since the lifetime of BSMs is primarliy defined by the ageing rate of a hottest cell in the string, thermal imbalance will result in premature death of battery pack. It should also be noted that the temperature of $\mathrm{Cell}_{i}$ in the $\mathrm{BSM}$ is coupled to temperatures of all upstream cells $\left(\mathrm{Cell}_{0}\right.$ to $\left.\mathrm{Cell}_{i-1}\right)$. Thus, if any single upstream cell in the BSM is in bad thermal condition then it will also affect temperature dynamics of all downstream cells. Therefore, one thermally exposed cell can reduce the lifetime of all downstream cells in the string. It has been reported in [11] that the temperature gradient in $\mathrm{xEV}$ battery packs is not negligible. Thus thermal balancing, in addition to SOC balancing, of xEV packs is necessary to enhance their lifetime.

\section{SOC BALANCING}

SOC balancing is one of the most important function of any advanced battery management system especially for long series string of cells. It improves not only the non-uniform ageing but also the total capacity of the BSM. The significance of cell SOC balancing in large BSMs has been studied thoroughly in the literature, see [14], [23], [24], [25]. The SOC balancer requires an external circuit to interact with each cell in a string. The external circuit can be dynamically reconfigured to provide the dissipative or non-dissipative alternate paths for direct energy flow between various cells in a string. In the following, we will discuss two main types of SOC balancers.

\section{A. Passive Cell Balancers (Dissipative)}

The passive balancer achieves cell equalization either by over-charging or by burning in shunt-resistors the excess charge of cells. It equalizes the SOC among cells only once, commonly at fully charged state of the BSM, during a chargedischarge cycle [21], [25]. Since the passive balancing device at most consists of only resistors, it can not be actively controlled externally. Thus, it does not require any complicated control algorithm except charge control. However, it is dissipative and is therefore less efficient. Moreover, cells of the passively balanced BSM are not equally utilized over whole charge-discharge cycle. Since cells cycle at different DODs, they suffer from non-uniform ageing [19]. Note that this method can only be used for lead-acid and NiMH batteries due to their tolerance against over-charge conditions [12], [14].

\section{B. Active Cell Balancers (Non-Dissipative)}

The active cell balancers use external switched circuits to actively transfer (shuttle, shuffle, shunt, or redistribute) the energy among cells of a BSM to achieve SOC balancing. The active balancing network commonly consists of switches and energy storage elements, like capacitors and inductors, which provide alternate paths for energy flow. Therefore, active cell balancers are highly energy efficient due to their non-dissipative nature and they can also be actively controlled using an external controller. However, the active cell balancers generally require more advanced control algorithms which become quite complex for large battery packs. The active cell balancer is the only viable solution for a lithium-ion packs because they cannot tolerate over-charging based passive balancing method [12], [14], [26]. There are various active balancing methods like cell shunting, cell-to-cell, cell-to-pack, pack-to-cell and cell-to-pack-to-cell, see [12], [27] for further details on balancing hardware and see [28] for optimizationbased thorough performance evaluation of various balancing methods. The capacity of an ideal actively balanced BSM is always given by the mean value of the cell capacities [19] as all cells are equally utilized in terms of their DODs. Therefore, the actively balanced BSM is able to deliver relatively higher Ahthroughput before its end-of-life. Thus, in order to maximize the capacity and to decelerate the ageing of the BSM, the use of an active balancer is desirable [19].

\section{THERMAL BALANCING}

The purpose of thermal balancing is to reduce the highest cell ageing rate in the BSM at the cost of slightly increasing the temperature (and hence the ageing rates) of other cells. In simple terms, the load of the weakest link in the whole chain is shared by other cells. Thermal imbalance in large battery packs can potentially be mitigated using following two approaches.

\section{A. Active Cooling with Reciprocating Coolant Flow}

Unidirectional coolant flow (UF) is commonly used in battery packs. However, this cooling scheme cannot compensate thermal imbalance due to temperature gradient in the coolant as shown in equation (7). Reciprocating coolant flow (RF) scheme has been suggested in [10] to solve this issue. In this scheme, the coolant flows back and forth in the battery pack at a fixed reciprocating time period. However, RF cannot solve imbalance arising from variation in cell resistance or variation in its other parameters like thermal resistance, see [18] for detailed performance evaluation of RF under parameter variations.

\section{B. Loss Balancing using Load Scheduling}

Thermal balancing can also be achieved by balancing losses among cells using load scheduling. In this method, each cell in the string is used according to its thermal condition. Thus, it has a full potential to compensate thermal imbalance due to both coolant temperature gradient and parametric variations. However, this requires a special hardware, which should

- be modular and distributes the BSM into $n$ cells.

- enable the bypassing of the load current around each cell.

- allow non-uniform use of cells. 


\section{Simultaneous Thermal And SOC Balancing}

The last section highlighted the importance of thermal and SOC balancing for enhancing the lifetime and usable capacity of a BSM. Instead of using separate thermal and SOC balancers, we are interested in achieving these two objectives simultaneously using a single active balancer based on load sharing concept.

\section{A. Simultaneous Balancing using Model-based Control}

Electro-thermal Model: Let us consider a lithium-ion BSM that consists of $n$ cells connected in series. The electrical dynamics of cells can be modeled using various equivalent circuit approaches [29] and thermal dynamics can be modeled using lumped capacitance and flow network modeling approach [10]. For instance, a simple electro-thermal model of any Cell ${ }_{i}$ in the BSM is given by (see [18] for modeling details)

$$
\begin{aligned}
\dot{T}_{s i}(t) & =a_{t i 1} T_{s 1}+\cdots+a_{t i i} T_{s i}+b_{s i} i_{B i}^{2}(t)+b_{t i} T_{f 0} \\
\dot{\xi}_{i}(t) & =-\frac{1}{3600 C_{i}} i_{B i}(t) \\
v_{B i}(t) & =v_{o c i}(t)-R_{s i} i_{B i}(t)
\end{aligned}
$$

where $a_{t i j}$ and $b_{t i}$ are thermal circuit parameters, $C_{i}$ is the capacity of $\operatorname{Cell}_{i}, b_{s i}=\frac{R_{s i}}{C_{s i}}$ where $R_{s i}$ and $C_{s i}$ are internal resistance and heat capacity of $\mathrm{Cell}_{i}$ respectively, and $T_{f 0}$ is the constant fluid temperature at the inlet. Temperature, $T_{s i}(t)$, and normalized SOC, $\xi_{i}(t)$, are states, terminal voltage $v_{B i}(t)$ is the output in response to the input current $i_{B i}(t)$, and $v_{o c i}(t)$ is an open circuit voltage of $\operatorname{Cell}_{i}$.

Remark 1. Note that $v_{o c i}(t)$ is generally a function of SOC, but it is assumed constant in this study. This approximation is justified if we assume battery operation in a certain SOC window. Also note that any $\mathrm{Cell}_{i}$ is in discharging mode when $i_{B i}(t)>0$ and in charging mode when $i_{B i}(t)<0$.

Control Mechanism: The BSM develops thermal and SOC imbalance among its cells due to variations in cell resistances $\left(R_{s i} \neq R_{s j}, \forall i, j\right)$ and cell capacities $\left(C_{i} \neq C_{j}, \forall i, j\right)$ respectively. The imbalance can potentially be mitigated using $i_{B i}(t)$ as an independent manipulating variable for each $\mathrm{Cell}_{i}$. However, to achieve this, we need some external control mechanism to manipulate $i_{B i}(t)$. In other words, we need a mechanism which should be able to independently adjust current of each $\mathrm{Cell}_{i}$ as follows

$$
i_{B i}(t)=u_{i}(t) i_{L}(t)
$$

where $u_{i}(t) \in[0,1]$ is a control knob (or duty cycle) of Cell $\mathrm{C}_{i}$ provided by an external control hardware and $i_{L}(t)$ is a load current. Since $i_{L}(t)$ is a given quantity at each time instant, the duty cycle $u_{i}(t)$ is used to adjust the cell current. Thus, to achieve thermal and SOC equalization, the duty cycle for each $\mathrm{Cell}_{i}$ should be given by

$$
u_{i}(t)=\kappa\left(\left\{\delta T_{s i j}(t)\right\}_{j=1}^{n},\left\{\delta \xi_{i j}(t)\right\}_{j=1}^{n}, v_{L d}(t)\right),
$$

where $\delta T_{s i j}$ and $\delta \xi_{i j}$ denotes temperature and SOC imbalance respectively between $\mathrm{Cell}_{i}$ and each $\mathrm{Cell}_{j}$ and $v_{L d}(t)$ is a load voltage demanded from BSM at each time instant.
Feasibility of Simultaneous Balancing and Requirements: Ideally, to achieve simultaneous thermal and SOC balancing at each time instant under all conditions, we need independent control variables for thermal and SOC dynamics. However, it should be noted that under current control framework $\left(i_{B i}(t) i_{B j}(t) \geq 0, \forall t\right)$ using equations (11)-(12), we cannot instantaneously control SOC and temperature independent of each other because $u_{i}(t)$ directly affects both states at each time instant, for example during discharging: $i_{B i}(t)>$ $i_{B j}(t) \Leftrightarrow i_{B i}^{2}(t)>i_{B j}^{2}(t)$. Moreover, cell temperature always increases whether we charge (increasing the SOC) or discharge (decreasing the SOC) the cell. Thus, thermal and SOC balancing are two tightly coupled and somewhat conflicting objectives and a natural question arises whether it is always feasible to achieve both of these objectives simultaneously.

Since it is not possible to have an independent control of temperature and SOC instantaneously. Therefore, to answer the above question, let us consider an alternative notion of temperature and SOC control in average sense. In this context, let us define average and rms currents of each $\mathrm{Cell}_{i}$

$$
I_{B a i}=\frac{1}{\delta t} \int_{t_{1}}^{t_{2}} i_{B i}(\tau) \mathrm{d} \tau, \quad I_{B r i}=\sqrt{\frac{1}{\delta t} \int_{t_{1}}^{t_{2}} i_{B i}^{2}(\tau) \mathrm{d} \tau}
$$

over a certain period $\delta t=t_{2}-t_{1}$ of a drive cycle of length $T_{D}$. Now suppose that the segment $\delta t$ of the drive cycle contains series of high and low current phases. Since temperature dynamics is quadratic in input current (i.e. $i_{B i}^{2}(t)$ ) and SOC is linear in input current $i_{B i}(t)$, it suggests that by appropriately scheduling the electrical load (current) of each cell during $\delta t$, it is possible to independently adjust $I_{B a i}$ and $I_{B r i}$ for each $\mathrm{Cell}_{i}$. This makes it possible to set $I_{B a i}>I_{B a j}$ and $I_{B r i}^{2}<I_{B r j}^{2}$ or $I_{B a i}<I_{B a j}$ and $I_{B r i}^{2}>I_{B r j}^{2}$ for any Cell ${ }_{i}$ and $\mathrm{Cell}_{j}$, enabling the independent control of their average temperature and SOC during $\delta t$. Under this notion, it is possible to achieve simultaneous balancing in average sense. Thus, a load profile with sufficient variations containing series of long low-current and short high-current phases is desirable in this regard. This kind of load profile is quite typical for $\mathrm{xEVs}$ due to natural variations (arise from topographical variations) in their speed and acceleration. If future load current can be predicted, then simultaneous thermal and SOC balancer can schedule the load for each cell using information about high and low current (including brake regeneration) phases in the drive cycle, cell resistances, temperature and SOCs.

However, to realize this goal we need an active balancing device with a special underlying modular structure that gives us sufficient degree-of-freedom to independently control each cell (or module) in a BSM (or battery pack). We may also need a state/parameter estimator and load predictor. In addition, we also need sufficient cell redundancy in the BSM i.e. the maximum BSM terminal voltage should be sufficiently greater than the maximum possible demanded load voltage, $v_{L d}(t)$, at any time instant.

Remark 2. It needs to be emphasized here that the simultaneous thermal and SOC balancing does not aim at perfect equalization of SOC and temperature all the time. After the initial balancing period of a highly unbalanced BSM, the 
perfect equalization of only SOC is needed at the boundaries (start and end) of a charge/discharge phase. However, inside the boundaries, the only objective is to keep both temperature and SOC deviations within certain reasonable limits, providing SOC and temperature deviations allowance during run time.

\section{B. Thermal and SOC Balancing as Optimization Problem}

It may be possible, though not obvious, to apply PID or LQ (linear quadratic) control framework to solve simultaneous thermal and SOC balancing problem. However, it seems more natural to apply predictive control framework due to large influence of future load currents on scheduling the load of each cell at present time. Thus, we need an intelligent control policy which uses accessible predictive (future) information to decide duty cycle of each cell at each sampling instant. We propose to formulate this problem as a convex optimization problem, which in words is stated below.

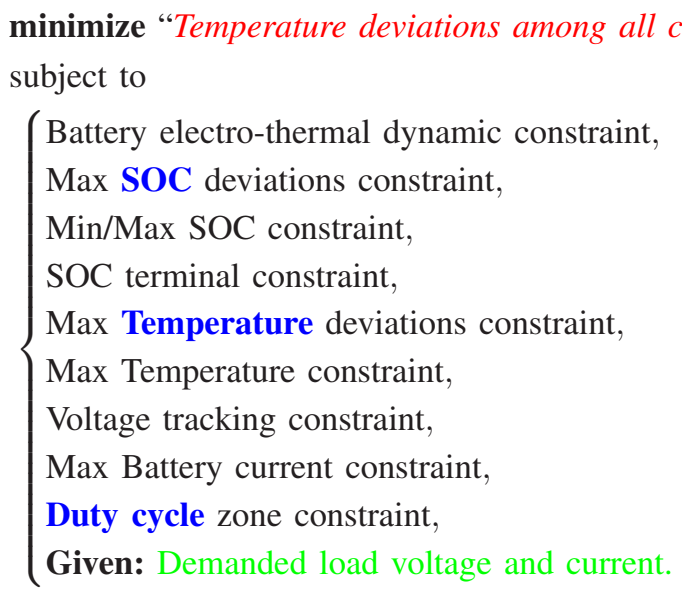

for all time instances over a prediction horizon $N_{p}$ with optimization variables SOC $\left(\xi_{i}(t)\right)$, temperature $\left(T_{s i}(t)\right)$, and duty cycle $u_{i}(t)$ of each Cell ${ }_{i}$. The variable $u_{i}(t)$ act as control knob of $\mathrm{Cell}_{i}$ and is provided by the balancing hardware. The output of this optimization problem over whole drive cycle of discrete time length $N_{D}$ will be the sequence of vectors $\{\boldsymbol{u}(t)\}_{t=1}^{N_{D}}$ where each $\boldsymbol{u}(t) \in \mathbb{R}^{n}$ consists of optimal duty cycles $u_{i}(t)$ for each $\operatorname{Cell}_{i}$. See [17], [18], and [19] for details.

\section{MLC as Thermal and SOC Balancer}

The previous sections highlighted the need of a special hardware technology with modular architecture to achieve the tightly coupled objectives of thermal and SOC balancing. In this subsection, we will briefly introduce one such technology - the cascaded H-bridge (CHB) multilevel converter (MLC) as shown in Fig. 3-which provides us enough degree-of-freedom to control the BSM (or battery pack) at cell (or module) level to achieve our set goals. The MLC provides a large redundancy in synthesizing the output voltage, which gives extra degree-of-freedom in control. The MLC, consisting of $n$ power cells (PCs), enables to distribute and modularize the BSM into smaller cells. Thus, the MLC cannot only act as a motor driver to generate a smoother output voltage waveform for electric machine ( $\mathrm{dc}$ machine here), but it can also act as a balancer to simultaneously control the modularized and distributed BSM on a cell level, see [17], [18], and [19] for

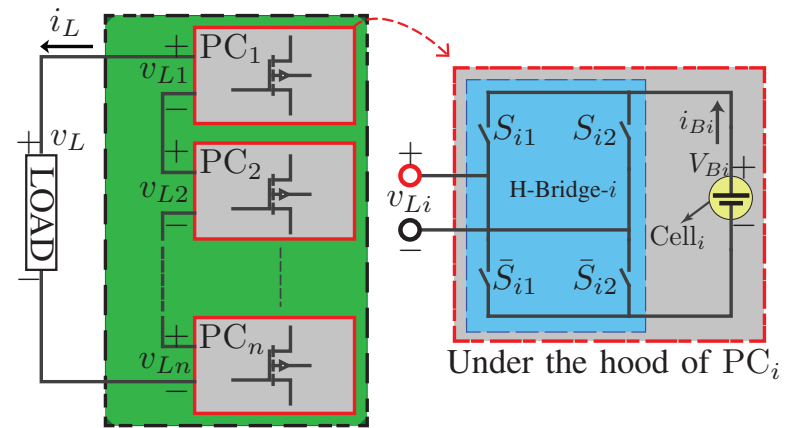

Fig. 3. Block diagram of a single-phase MLC. Note that the BSM is distributed as cells among $n$ PCs and load is a dc machine here.

further details on using MLC as an integrated cell balancer and motor driver.

\section{Simulation EXAmple}

\section{A. Simulation Setup}

The following simulation study is based on the solution of problem (14). To solve this problem, we used CVX, a MATLAB-based package for specifying and solving convex programs using disciplined convex programming ruleset, see [30] and [31]. CVX transforms MATLAB ${ }^{\circledR}$ into a modeling language, allowing constraints and objectives to be specified using standard MATLAB ${ }^{\circledR}$ expression syntax.

Before presenting simulation results, let us define timevarying dischargeable capacity of each $\mathrm{Cell}_{i}$ and the BSM as follows

$$
C_{i, D}(t)=\xi_{i}(t) C_{i}, \quad C_{\mathrm{BSM}, D}(t)=\xi_{\mathrm{BSM}}(t) C_{\mathrm{BSM}}(t)
$$

where $\xi_{\mathrm{BSM}}(t)$ and $C_{\mathrm{BSM}}(t)$ are SOC and time-varying total capacity of BSM given by equations (3) and (4) respectively. Now let us consider a BSM consisting of two cells having capacity and resistance variations $\left(C_{2}-C_{1} \neq 0\right.$ and $R_{s 2}-R_{s 1} \neq 0$ ). It is assumed that the difference in initial dischargeable capacity of two cells is non-zero as well $\left(C_{2, D}(0)-C_{1, D}(0) \neq 0\right)$. We have four possible cases in this example:

- Case-1: $C_{1, D}(0)<C_{2, D}(0)$ and $R_{s 1}<R_{s 2}$,

- Case-2: $C_{1, D}(0)>C_{2, D}(0)$ and $R_{s 1}<R_{s 2}$,

- Case-3: $C_{1, D}(0)<C_{2, D}(0)$ and $R_{s 1}>R_{s 2}$,

- Case-4: $C_{1, D}(0)>C_{2, D}(0)$ and $R_{s 1}>R_{s 2}$.

The objective is to simultaneously achieve thermal and SOC balancing while meeting at the same time the demand (voltage $v_{L d}(t)$ ) of a dc machine. Note that the drive cycle data in terms of $v_{L d}(t)$ is shown as dotted green line in Figures 4 and 5 .

Remark 3. Since the demanded machine speed is proportional to $v_{L d}(t)$, variations in $v_{L d}(t)$ implies variations in load profile. Note series of acceleration and deceleration periods inside each driving phase marked as dotted pink circle as shown in figure 5(b). These driving phases can be exploited to achieve simultaneous thermal and SOC balancing.

Remark 4. Note that in the following simulation results, all cells in the series string are assumed to have same polarity all the time. It implies that all cell currents $\left(i_{B i}(t): \mathrm{s}\right.$ for all Cell $\left.{ }_{i}\right)$ always have the same $\operatorname{sign}\left(i_{B i}(t) i_{B j}(t) \geq 0, \forall i, j, \forall t\right)$. 


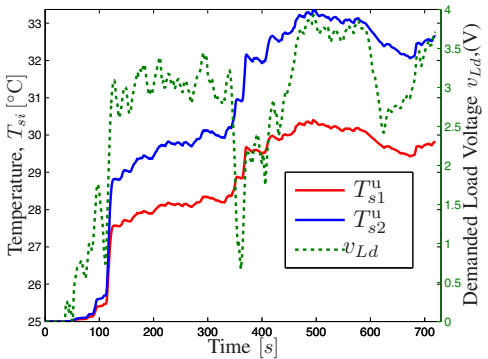

(a) Temperature of each cell under uniform use $\left(i_{B r 1}^{2}(t)=i_{B r 2}^{2}(t)\right)$.

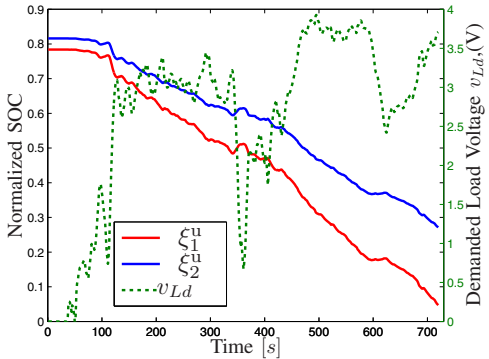

(b) SOC of each cell under uniform use $\left(i_{B 1}(t)=i_{B 2}(t)\right)$.

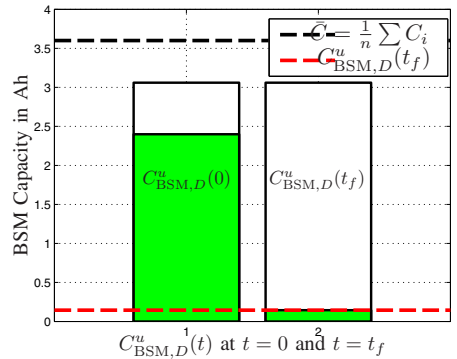

(c) Dischargeable BSM capacity shown in green at start and end of discharging.

Fig. 4. Simulation results for uniform use of all cells in the BSM. Note that $C_{2}>C_{1}, \xi_{2}(0)>\xi_{1}(0), R_{s 2}>R_{s 1}$, and $v_{L d}$ is demanded load voltage.

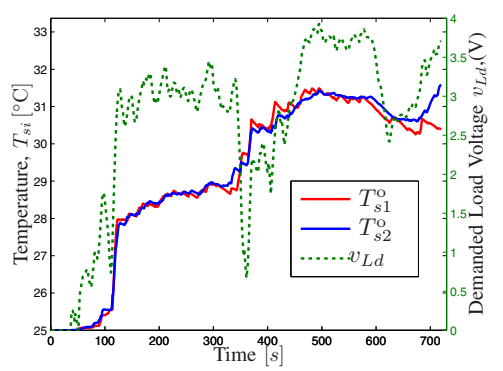

(a) Temperature of each cell under nonuniform use with demanded load voltage $v_{L d}(t)$ shown in dotted green.

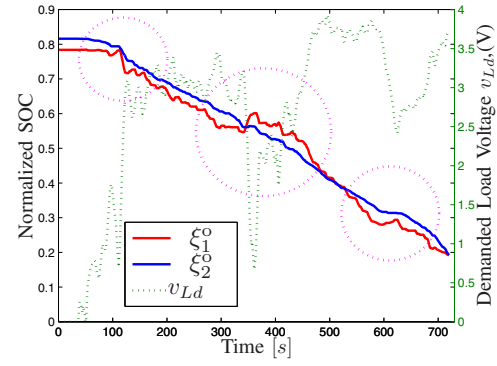

(b) SOC of each cell along with load voltage $v_{L d}(t)$ shown in dotted green and dotted circles denote drive phases with load variations.

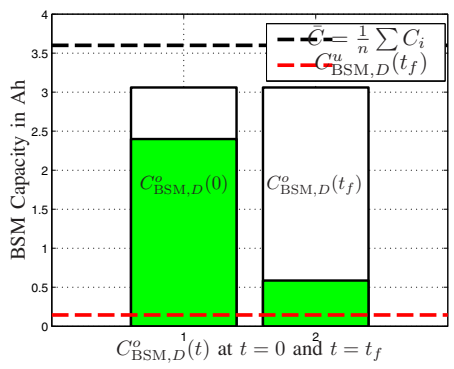

(c) Dischargeable BSM capacity shown in green at start and end of discharging.

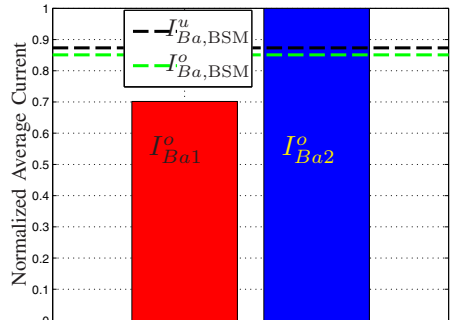

Cell Number, Cell

(d) Normalized average cell current over whole drive cycle. Note $I_{B a 2}>I_{B a 1}$.

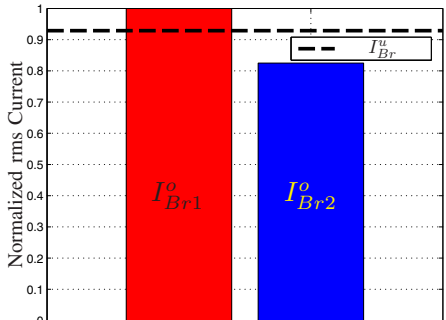

Cell Number, $\mathrm{Cell}_{i}^{2}$

(e) Normalized rms current over whole drive cycle. Note $I_{B r 2}^{2}<I_{B r 1}^{2}$.

Fig. 5. Simulation results for non-uniform use $\left(I_{B a 1} \neq I_{B a 2}\right.$ and $\left.I_{B r 1}^{2} \neq I_{B r 2}^{2}\right)$ of cells in the BSM. Note that $C_{2}>C_{1}, \xi_{2}(0)>\xi_{1}(0)$, and $R_{s 2}>R_{s 1}$. Compare figures 4 and 5 and note the difference in temperature and SOC evolution of cells and final dischargeable capacities of the BSM under uniform and non-uniform use.

\section{B. Uniform Use of Cells}

Thermal and SOC imbalance will obviously increase further with time for all cases if both cells are uniformly used. Figure 4 shows this effect particularly for case-1. Figures 4(a) and 4(b) show evolution of temperature and SOC of each cell respectively and figure 4(c) shows dischargeable capacity $C_{\mathrm{BSM}, D}^{u}(t)$ of BSM at initial and final times of drive cycle.

\section{Non-uniform Use of Cells}

Since two cells have nonuniform characteristics, the optimal policy is to also use them non-uniformly according to their temperature and SOC. Note that case- 2 can be easily handled by using Cell $1_{1}$ more $\left(I_{B a 1}>I_{B a 2}\right.$ and $\left.I_{B r 1}>I_{B r 2}\right)$ and similarly case- 3 by using Cell 1 less compared to $\mathrm{Cell}_{2}$. However, we have conflicting situation in cases 1 and 4. For example, in case-1, SOC balancing requires $\mathrm{Cell}_{1}$ to be used less than $\mathrm{Cell}_{2}$ but thermal balancing requires $\mathrm{Cell}_{1}$ to be used more than $\mathrm{Cell}_{2}$ and vice versa in case-4. In the following, case- 1 is analyzed in detail through a simulation study.

Simulation results for case-1 under non-uniform use are shown in Figure 5. Figures 5(a) and 5(b) show evolution of temperature and SOC of each cell respectively, figure 5(c) shows dischargeable capacity $C_{\mathrm{BSM}, D}^{o}(t)$ of BSM at initial and final times of drive cycle, and figures 5(d) and 5(e) show normalized average and rms current respectively of each cell. These figures show that thermal balancing can be achieved by using (higher resistance) Cell $_{2}$ less than Cell $_{1}$ during short high current phases (either charging or discharging) of the drive cycle. This leads to relatively quick increase in temperature of $\mathrm{Cell}_{1}$ compared to that of $\mathrm{Cell}_{2}$ as losses are quadratic in current. The SOC balancing is achieved simultaneously by using $\mathrm{Cell}_{2}$ more than $\mathrm{Cell}_{1}$ during long low 
current phases. This scheme of cell usage results in $I_{B a 2}>$ $I_{B a 1}$ (for SOC balancing) and $I_{B r 2}<I_{B r 1}$ (for thermal balancing) as shown in figures 5(d) and 5(e) respectively. Note (inside pink dotted circles shown in Figure 5(b)) that (lower resistance) Cell $_{1}$ absorbs most of the charging energy during driving phases of high regenerative braking and delivers most of the energy during following high acceleration phases. It should be noticed that a certain level of SOC and temperature deviation allowance also facilitates achieving the objective of simultaneous balancing. Also, compare figures 4(c) and 5(c) and note the difference in final dischargeable capacities $\left(C_{\mathrm{BSM}, D}^{o}\left(t_{f}\right)>C_{\mathrm{BSM}, D}^{u}\left(t_{f}\right)\right)$ of the BSM under uniform and nonuniform use of cells. It shows that, compared to a uniformly used (unbalanced) BSM, a non-uniformly used (balanced) BSM may provide higher ampere-hour throughput during each charge/discharge cycle. Note that case 4 can similarly be handled by using Cell $_{1}$ less during high current phases and more during low current intervals.

\section{CONCLUSIONS}

We have reviewed simultaneous thermal and SOC balancing and added more insight into our previous studies of this problem. It is shown, through simple arguments and a simulation based case study, that this problem can be formulated and solved in a predictive control framework by optimally

- scheduling the electrical load of each cell,

- redistributing the power losses among cells as per the level of temperature and SOC imbalance,

- exploiting the brake regeneration phases and variations in the load profile,

- exploiting the SOC and temperature deviation allowance,

- exploiting the cell redundancy in the battery pack.

However, for this we need load predictions over a reasonable horizon. We also need an active balancing device, like MLC, with a very special underlying modular structure that gives us sufficient degree-of-freedom to independently control each cell (or module) in a BSM (or battery pack).

\section{REFERENCES}

[1] A. Barré, B. Deguilhem, S. Grolleau, M. Gérard, F. Suard, and D. Riu, "A review on lithium-ion battery ageing mechanisms and estimations for automotive applications," Journal of Power Sources, vol. 241, no. 0, pp. $680-689,2013$.

[2] M. Broussely, P. Biensan, F. Bonhomme, P. Blanchard, S. Herreyre, K. Nechev, and R. Staniewicz, "Main aging mechanisms in li ion batteries," Journal of Power Sources, vol. 146, no. 1, pp. 90-96, 2005.

[3] J. Vetter, P. Novak, M. Wagner, C. Veit, K.-C. Möller, J. Besenhard, M. Winter, M. Wohlfahrt-Mehrens, C. Vogler, and A. Hammouche, "Ageing mechanisms in lithium-ion batteries," Journal of power sources, vol. 147 , no. 1, pp. 269-281, 2005.

[4] P. Ramadass, B. Haran, P. M. Gomadam, R. White, and B. N. Popov, "Development of first principles capacity fade model for li-ion cells," Journal of The Electrochemical Society, vol. 151, no. 2, pp. A196-A203, 2004.

[5] P. Ramadass, B. Haran, R. White, and B. N. Popov, "Mathematical modeling of the capacity fade of li-ion cells," Journal of Power Sources, vol. 123, no. 2, pp. 230-240, 2003.

[6] J. Wang, P. Liu, J. Hicks-Garner, E. Sherman, S. Soukiazian, M. Verbrugge, H. Tataria, J. Musser, and P. Finamore, "Cycle-life model for graphite-LiFePO 4 cells," Journal of Power Sources, vol. 196, no. 8, pp. 3942-3948, 2011

[7] S. Paul, C. Diegelmann, H. Kabza, and W. Tillmetz, "Analysis of ageing inhomogeneities in lithium-ion battery systems," Journal of Power Sources, 2013.
[8] T. M. Bandhauer, S. Garimella, and T. F. Fuller, "A critical review of thermal issues in lithium-ion batteries," Journal of the Electrochemical Society, vol. 158, no. 3, pp. R1-R25, 2011.

[9] B. Wu, V. Yufit, M. Marinescu, G. J. Offer, R. F. Martinez-Botas, and N. P. Brandon, "Coupled thermal-electrochemical modelling of uneven heat generation in lithium-ion battery packs," Journal of Power Sources, 2013.

[10] R. Mahamud and C. Park, "Reciprocating air flow for li-ion battery thermal management to improve temperature uniformity," Journal of Power Sources, vol. 196, no. 13, pp. 5685 - 5696, 2011.

[11] Y. Troxler, B. Wu, M. Marinescu, V. Yufit, Y. Patel, A. J. Marquis, N. P. Brandon, and G. J. Offer, "The effect of thermal gradients on the performance of lithium-ion batteries," Journal of Power Sources, vol. 247, pp. 1018-1025, 2014

[12] J. Gallardo-Lozano, E. Romero-Cadaval, M. I. Milanes-Montero, and M. A. Guerrero-Martinez, "Battery equalization active methods," Journal of Power Sources, vol. 246, pp. 934-949, 2014.

[13] W. C. Lee, D. Drury, and P. Mellor, "Comparison of passive cell balancing and active cell balancing for automotive batteries," in Vehicle Power and Propulsion Conference (VPPC), 2011 IEEE, sept. 2011, pp. $1-7$

[14] J. Cao, N. Schofield, and A. Emadi, "Battery balancing methods: A comprehensive review," in Vehicle Power and Propulsion Conference, 2008. VPPC '08. IEEE, sept. 2008, pp. $1-6$.

[15] W. Bentley, "Cell balancing considerations for lithium-ion battery systems," in Battery Conference on Applications and Advances, 1997., 12th Annual, jan 1997, pp. 223 -226.

[16] P. Krein, "Battery management for maximum performance in plugin electric and hybrid vehicles," in Vehicle Power and Propulsion Conference, 2007. VPPC 2007. IEEE, sept. 2007, pp. 2 -5.

[17] F. Altaf, L. Johannesson, and B. Egardt, "Evaluating the potential for cell balancing using a cascaded multi-level converter using convex optimization," in IFAC Workshop on Engine and Powertrain Control, Simulation and Modeling, 2012, Oct. 2012.

[18] — , "On thermal and state-of-charge balancing using cascaded multilevel converters," Journal of Power Electronics, vol. 13, no. 4, pp. 569583,2013

[19] F. Altaf, Thermal and State-of-Charge Balancing of Batteries using Multilevel Converters. Licentiate Thesis, Chalmers University of Technology, 2014.

[20] L. Lu, X. Han, J. Li, J. Hua, and M. Ouyang, "A review on the key issues for lithium-ion battery management in electric vehicles," Journal of Power Sources, vol. 226, pp. 272-288, 2013.

[21] L. Zhong, C. Zhang, Y. He, and Z. Chen, "A method for the estimation of the battery pack state of charge based on in-pack cells uniformity analysis," Applied Energy, vol. 113, pp. 558-564, 2014.

[22] Y. Zheng, L. Lu, X. Han, J. Li, and M. Ouyang, "Lifepo4 battery pack capacity estimation for electric vehicles based on charging cell voltage curve transformation," Journal of Power Sources, 2013.

[23] W. Bentley, "Cell balancing considerations for lithium-ion battery systems," in Battery Conference on Applications and Advances, 1997., Twelfth Annual. IEEE, 1997, pp. 223-226.

[24] P. A. Cassani and S. S. Williamson, "Significance of battery cell equalization and monitoring for practical commercialization of plug-in hybrid electric vehicles," in Applied Power Electronics Conference and Exposition, 2009. APEC 2009. Twenty-Fourth Annual IEEE. IEEE, 2009, pp. 465-471.

[25] D. Andrea, Battery Management Systems for Large Lithium Ion Battery Packs, 1st ed. Artech House, 92010.

[26] C.-H. Kim, M. young Kim, H. sun Park, and G.-W. Moon, "A modularized two-stage charge equalizer with cell selection switches for seriesconnected lithium-ion battery string in an hev," Power Electronics, IEEE Transactions on, vol. 27, no. 8, pp. 3764-3774, 2012.

[27] A. Manenti, A. Abba, A. Merati, S. Savaresi, and A. Geraci, "A new bms architecture based on cell redundancy," Industrial Electronics, IEEE Transactions on, vol. 58, no. 9, pp. 4314-4322, 2011.

[28] M. Preindl, C. Danielson, and F. Borrelli, "Performance evaluation of battery balancing hardware," in Control Conference (ECC), 2013 European. IEEE, 2013, pp. 4065-4070.

[29] X. Hu, S. Li, and H. Peng, "A comparative study of equivalent circuit models for li-ion batteries," Journal of Power Sources, vol. 198, no. 0, pp. $359-367,2012$

[30] M. Grant and S. Boyd, "CVX: Matlab software for disciplined convex programming, version 1.21," ../../cvx, Apr. 2011.

[31] S. Boyd and L. Vandenberghe, Convex Optimization. Cambridge University Press, 2006. 